\title{
Multichannel fiber laser Doppler vibrometer studies of low momentum and hypervelocity impacts
}

\author{
Julio E. Posada-Roman ${ }^{\mathrm{a}, *}$, David A. Jackson ${ }^{\mathrm{b}}$, Mike J. Cole ${ }^{\mathrm{b}}$, Jose A. Garcia-Souto \\ a Department of Electronics Technology, GOTL, Universidad Carlos III de Madrid, Butarque 15, 28911, Leganes, Madrid, Spain \\ ${ }^{\mathrm{b}}$ Applied Optics Group, School of Physical Sciences, University of Kent, Canterbury, Kent CT2 7NH, UK
}

\section{A R T I C L E I N F O}

\section{Keywords:}

Impacts

Vibration

Laser Doppler

Heterodyne

Light gas gun

\begin{abstract}
A B S T R A C T
A multichannel optical fiber laser Doppler vibrometer was demonstrated with the capability of making simultaneous non-contact measurements of impacts at 3 different locations. Two sets of measurements were performed, firstly using small ball bearings $(1 \mathrm{~mm}-5.5 \mathrm{~mm})$ falling under gravity and secondly using small projectiles $(1 \mathrm{~mm}$ ) fired from an extremely high velocity light gas gun (LGG) with speeds in the range $1 \mathrm{~km} / \mathrm{s}-$ $8 \mathrm{~km} / \mathrm{s}$. Determination of impact damage is important for industries such as aerospace, military and rail, where the effect of an impact on the structure can result in a major structural damage. To our knowledge the research reported here demonstrates the first trials of a multichannel fiber laser Doppler vibrometer being used to detect hypervelocity impacts.
\end{abstract}

\section{Introduction}

Impacts can be expected to occur during the lifetime of structures, for example impacts on aircraft due to shrapnel and other airborne particles. Hence the determination of impact damage is important for industries such as passenger aircraft manufactures and their operating companies. Different tests are used to simulate various types of impact: drop-weight tests simulate low-velocity impacts; air-gun systems, in which a small projectile is propelled at high speeds, simulate the type of impacts encountered during aircraft service (e.g. during takeoffs and landings) [1]. To generate extreme velocities (hypervelocity) such as those experienced by spacecraft a light gas gun is required, for example to simulate micrometeoroid impacts and checking shielding and tether arrangements.

Currently, the Light Gas Gun (LGG) at the University of Kent uses Polyvinylidene fluoride (PVDF) probes to monitor the tests and development of damage induced during impacts. These probes are mounted on the edge of the target loading it mechanically and affecting the dynamics of the impact [2,3]. In some cases PVDF probes are not compatible with the specific experimental requirements (e.g. tests on hot targets).

Previously we performed experiments with fiber Bragg gratings (FBG) inside the LGG. The gratings were mounted under tension on the target and interrogated as optical strain gauges. We reported the use of FBGs to measure impacts caused by small projectiles fired from the LGG [4]. This approach was shown to be very effective in comparison with the PVDF transducers [5-7]. This is because the FBG could be bonded much closer to the impact point of the projectile on the target thus providing more precise data on the dynamics of the impact [4]. Despite the good results there are problems with the displacement resolution $(1.24 \mu \mathrm{m})$ and requirement for the FBG to be mounted with a known strain. Moreover, the sensors cannot be readily removed from the target due to the strong adhesives used.

Target mounted probes can be damaged by the impact even when it is not directly hit by the hypervelocity particle. This is a significant problem when the probe is bonded to the target as is the case with both, PVDF probes and FBG sensors. Another problem with any contact sensor is that it has to be calibrated when installed on the target. The multichannel fiber laser Doppler vibrometer (MFLDV) subject of this paper does not suffer from any of these problems as there is no physical contact between the sensor and the probe and, equally important, the measurements are absolute. The system is ideally suited for complex impact tests such as those with ice or salt projectiles, which demand multiple channel simultaneous sensing.

We adopted the MFLDV previously described in [8] with miniature variable focus collimator probes inside the LGG to analyze the effects of impacts. The main advantages are that the measurements are noncontact, $1 \mathrm{~nm}$ displacement resolution is achievable, and the carrier frequency can be optimally selectable for digital processing. Both the amplitude variations generated by the impacts at different target locations and the relative phases between the channels can be determined. This data can be used in modelling the target dynamics.

\footnotetext{
* Corresponding author.

E-mail addresses: jposada@ing.uc3m.es (J.E. Posada-Roman), d.a.jackson@kent.ac.uk (D.A. Jackson), m.j.cole@kent.ac.uk (M.J. Cole), jsouto@ing.uc3m.es (J.A. Garcia-Souto).
} 


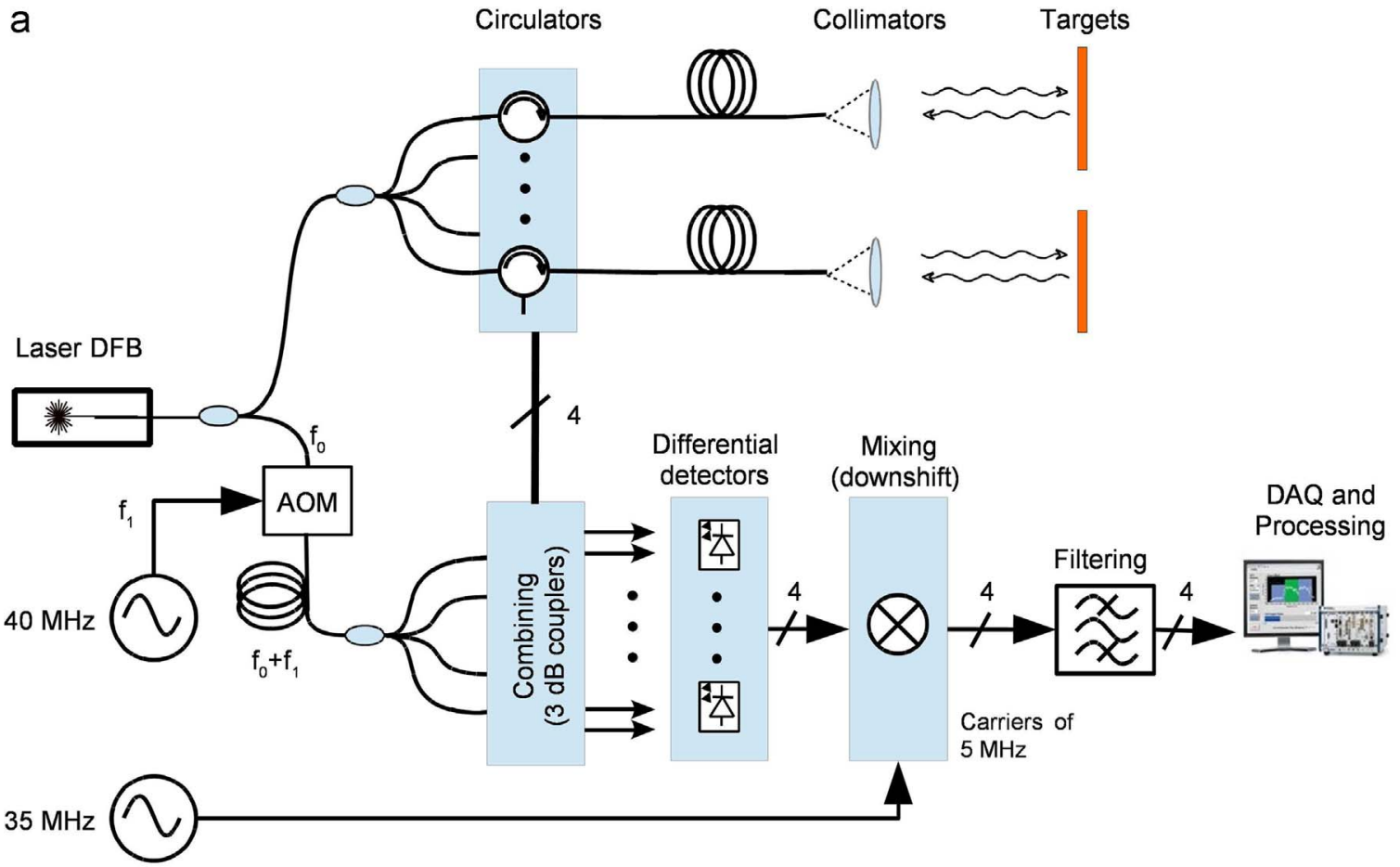

b

\section{4 channel power \\ distribution unit}

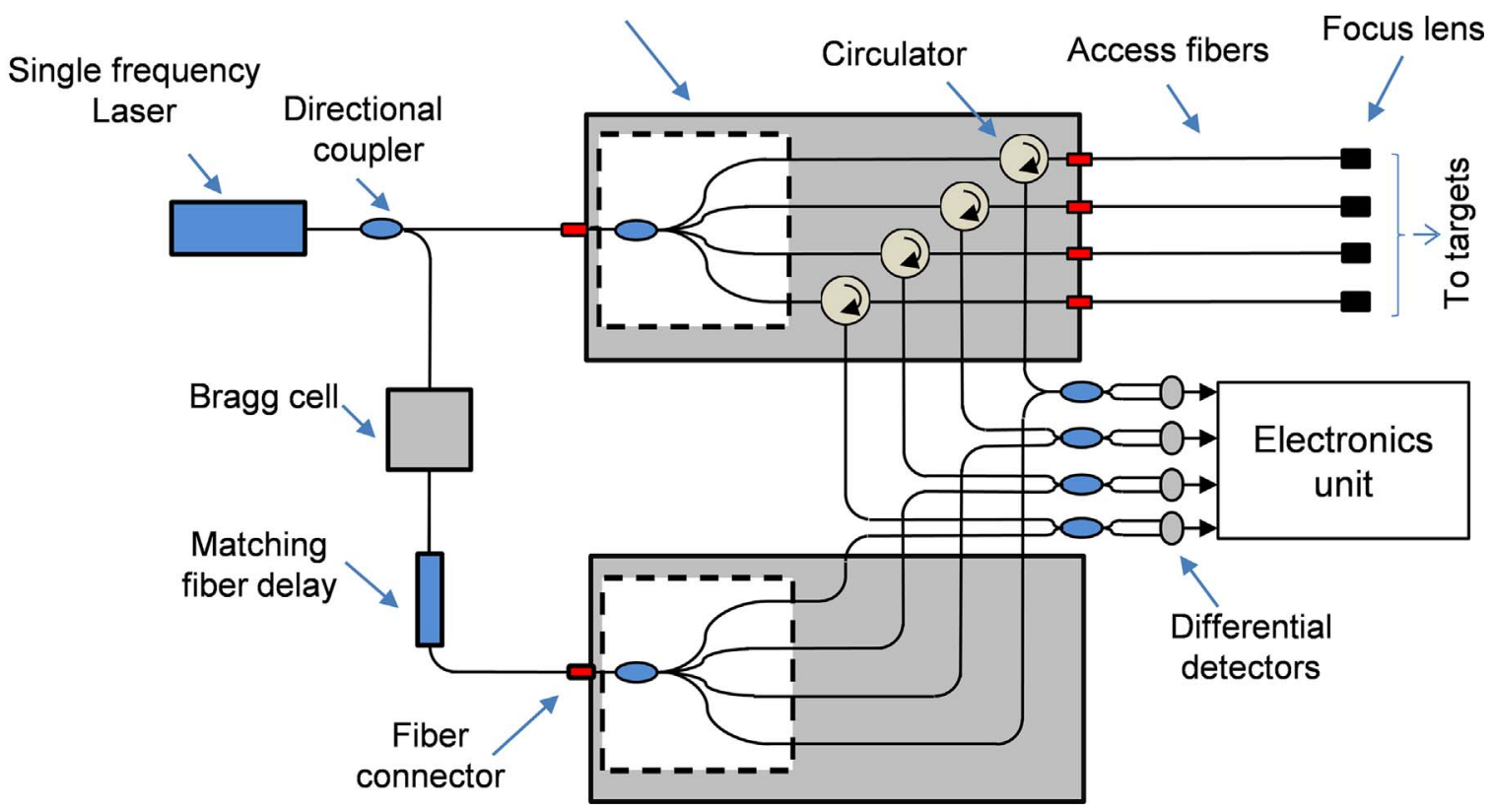

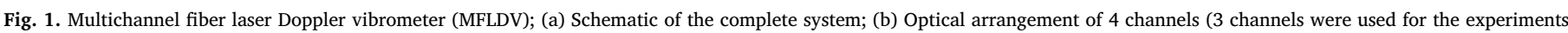
reported below).

To enhance the recovered signal amplitude a $1 \mathrm{~mm}$ diameter selfadhesive 3Ms reflective paper (effectively weightless) was positioned at each target location. A similar system for general use is reported in reference [9] which has a different topology to that described in Section 2 as it has two acousto-optic modulators (AOM), one a frequency shifter for the Doppler effect, the other a free spaced AOM which injects 4 differentially shifted beams into the transceiver links. Only one detector is required, but the experiments reported were relatively low speed impacts.

\section{Four channel heterodyne MFLDV}

Fig. 1 shows the MFLDV: the schematic of the complete system (Fig. 1a) and the optical arrangement of 4 channels (Fig. 1b), which is reported in detail in [8]. It contains two 1-4 channel power distribution units (Fig. 1b). Light from a single frequency $1550 \mathrm{~nm}$ laser diode is transferred to two paths by a $3 \mathrm{~dB}$ directional coupler. The light on one of the paths propagates to the targets via 4 circulators and hence to 4 variable focus collimators where the back reflected signals are transferred to the inputs of four $3 \mathrm{~dB}$ couplers. The optical power per 
channel to the target is $0.8 \mathrm{~mW}$ in this arrangement (Fig. 1b). The light propagating along the second path is frequency shifted by $40 \mathrm{MHz}$ with a fiberized Bragg cell (Fig. 1a) and then propagates through a matching fiber delay where it splits into 4 channels. It is then transferred to the other inputs of the $3 \mathrm{~dB}$ couplers and mixes with light back reflected from the targets (Fig. 1b). As only 3 channels could be deployed in the LGG due to space limitations in the LGG vacuum's seal, the fourth channel could possibly be used to continuously monitor the optical system especially before and after each impact.

The 3 heterodyne signals were detected with $125 \mathrm{MHz}$ bandwidth photodiodes. As these signals were at $40 \mathrm{MHz}$ each signal was down shifted to $5 \mathrm{MHz}$ using electronic mixers where the local oscillator was $35 \mathrm{MHz}$ (Fig. 1a) in order to match the specifications of the digital processing unit available. The relative phases among the channels are maintained by this topology which is typically used in optical metrology and radar. As the coherence length of the source used was very large, exact matching of the signal and reference paths was not necessary to obtain high contrast interference signals. The 3 simultaneously recovered optical heterodyned signals were digitized with a high speed DAQ and processed with a virtual instrument implemented in LabVIEW [10].

The resolution achieved by the system was determined using a new calibration method based on the Bessel function of the first kind [11]. It estimates the resolution from the signal to noise ratio of the detected heterodyne carrier. The minimum detectable displacement of the system was measured to be $0.7 \mathrm{~nm}$ (RMS) at a bandwidth of $90 \mathrm{kHz}$, i.e. $2 \mathrm{pm} / \sqrt{ } \mathrm{Hz}$. The system is able to measure vibration velocities up to $3.8 \mathrm{~m} / \mathrm{s}$ determined by the frequency of the $5 \mathrm{MHz}$ carrier.

\section{Portable test rig for impact studies}

Prior to the proposed study of the vibration induced in a target caused by hypervelocity impacts a complete mount with all the optical probes and fiber transceiver links was set up. This enabled the operation of the system to be tested with low momentum impacts. The mount was designed such that with minimum modifications it could be directly installed in the LGG.

The assembly shown in Fig. 2 is the mount used for both low velocity drop tests and Hypervelocity impacts. It consists of 2 plates, the top target plate made of carbon fiber (for the experiments reported here) and a second plate made of aluminum on which three MFLDV optical probes were mounted. These probes were mounted at $120^{\circ}$ allowing their radial positions to be adjusted such that vibrations at different diameters on the target could be determined. The plates were held together with 4 circular posts allowing the test facility to be located and operated in other areas. The variable focus collimators were focused on the bottom of the test plate at $\sim 10 \mathrm{~cm}$.

\section{Low momentum impact studies}

\subsection{Experimental set-up}

The impact signals were generated by dropping a range of small ball-bearings from a height of $10 \mathrm{~cm}$. Data collection was initiated using an optical gate triggered as the ball-bearing passed through a laser beam. Table 1 summarizes the characteristics of the ball bearings used in these tests.

The positions of the targets of the corresponding probes are shown in Fig. 3, which is a schematic view from the underside of the plate. Small discs of $3 \mathrm{M}$ reflective paper were mounted at each location. The impact distances from the center of the target are $30 \mathrm{~mm}, 8 \mathrm{~mm}$ and $40 \mathrm{~mm}$ for the target points $\mathrm{T} 1, \mathrm{~T} 2$ and $\mathrm{T} 3$ that correspond to the measurement channels $\mathrm{Ch} 1, \mathrm{Ch} 2$ and $\mathrm{Ch} 3$, respectively.

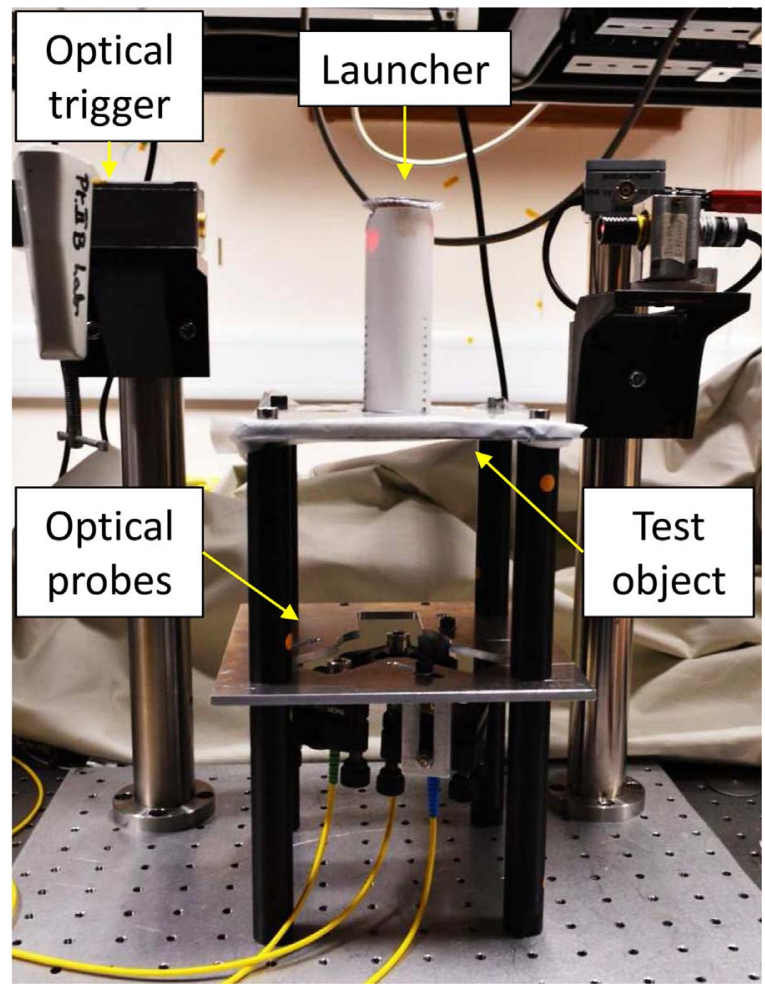

Fig. 2. Arrangement for studying low momentum impacts on a carbon fiber plate.

Table 1

Properties of the ball bearings used in the drop test.

\begin{tabular}{lll}
\hline Diameter $(\mathrm{mm})$ & Ball mass $(\mathrm{g})$ & Material \\
\hline 5.5 & 1.307 & Tungsten \\
5.0 & 0.988 & \\
4.5 & 0.7218 & \\
4.0 & 0.5083 & Steel \\
3.0 & 0.2161 & \\
2.5 & 0.0686 & \\
1.5 & 0.014183 & \\
1.0 & 0.004 & \\
\hline
\end{tabular}

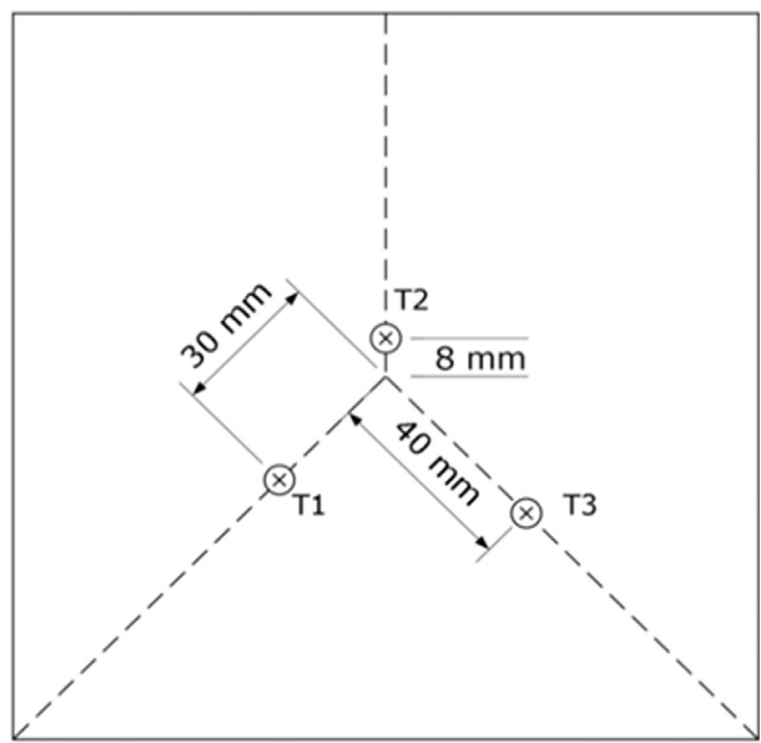

Fig. 3. Position of the targets on the rear face of the plate and their corresponding measurement channel: $\mathrm{T} 1=\mathrm{Ch} 1, \mathrm{~T} 2=\mathrm{Ch} 2, \mathrm{~T} 3=\mathrm{Ch} 3$. 


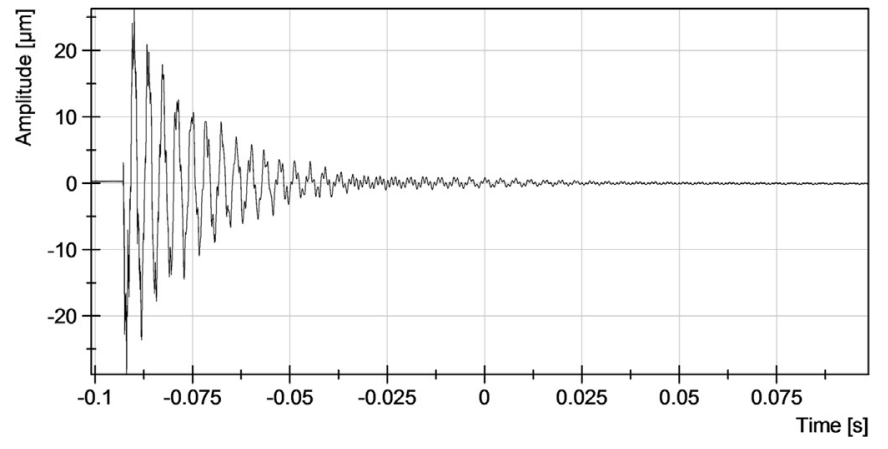

Fig. 4. Variation of the intensity at $\mathrm{T} 1 \mathrm{as}$ a function of time for a $0.0686 \mathrm{~g}$ weight drop.

\subsection{Results}

An example of the typical vibration signals registered during the low momentum tests is shown in Fig. 4. In this case, it corresponds to the vibration amplitude measured on the back face of the plate, at location $\mathrm{T} 1$, resulting from a $0.0686 \mathrm{~g}$ weight dropped on the center of the plate. The variation of the amplitude with time shows an exponential decay with a natural frequency $250.5 \mathrm{~Hz}$ which contains several harmonics. This can be observed in more detail in Fig. 5 where it is shown typical spectrum of the vibration signals during the drop test.

Similar spectral response, with the natural frequency around $250 \mathrm{~Hz}$, was observed in all the cases for the different momentum impacts. Fig. 6 shows the results of the different amplitudes of the displacement measured at the three locations $\mathrm{T} 1, \mathrm{~T} 2$ and $\mathrm{T} 3$, as a function of momentum. It can be observed in Fig. 6 that the displacement of the plate presents a linear behavior for the lower momentum impacts, which agrees with theoretical analysis of a vibrating clamped square plate [12]. The range of amplitudes measured during the test was from $3.2 \mu \mathrm{m}$ to $651 \mu \mathrm{m}$. The data in Figs. 4 and 6 demonstrate the extreme sensitivity that can be achieved with the MFLDV.

\section{Hypervelocity impact studies}

\subsection{Installation}

The two stage, LGG, (Fig. 7) used for this study was the same as reported in [4]. It can fire small projectiles (1 $\mathrm{mm}$ size) at speeds from $1 \mathrm{~km} / \mathrm{s}$ to $8.4 \mathrm{~km} / \mathrm{s}$. The speed of each shot is measured to $\sim 1 \%$ accuracy; the target chamber is evacuated to $\sim 0.5 \mathrm{mbar}$.

The target region of the LGG is shown in Fig. 8 with the mount containing the 3 probes and fiber transceivers. The mount shown in Fig. 2 is attached to the open-able rear port of the LGG. The only modifications to the optics required was the use of 3 addition fiber patch cords which were mounted in vacuum seals (Fig. 9) at one of the ports of the LGG. A thin plastic sheet is mounted in front of the collimators to protect them from the carbon fiber dust generated by the impact.

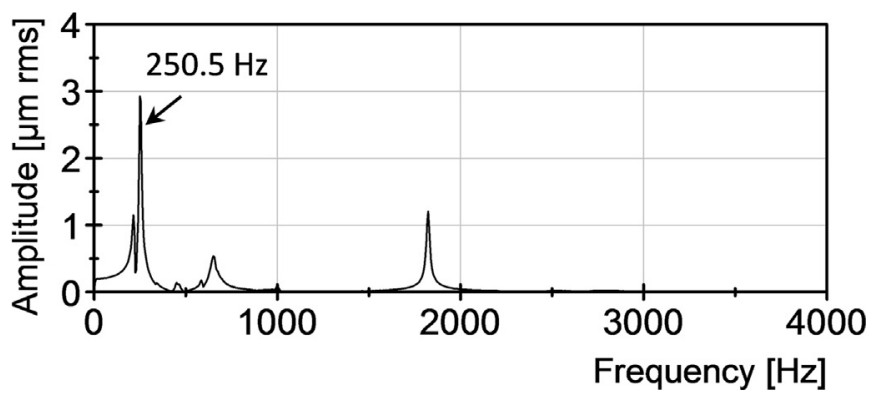

Fig. 5. Typical spectrum of the signals observed during the drop test.

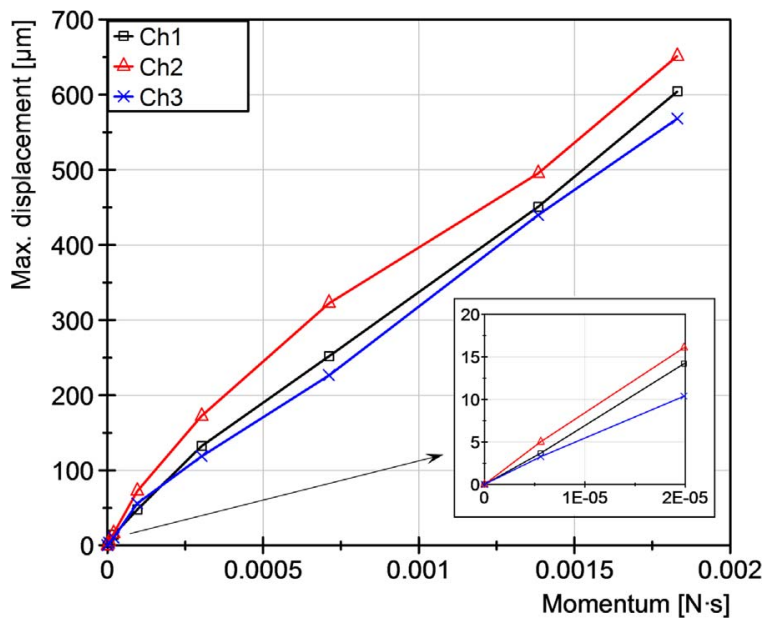

Fig. 6. Displacement of the surface of the plate as a function of impact momentum.

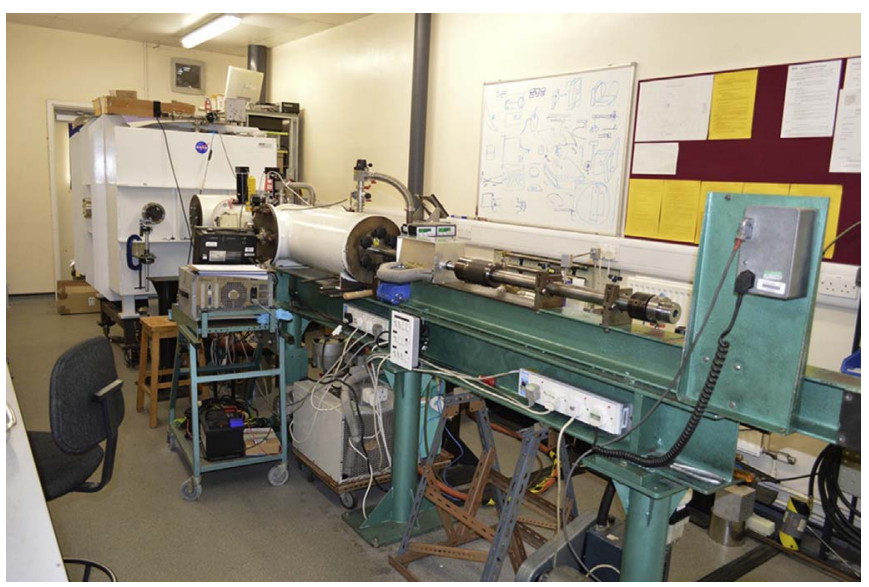

Fig. 7. The light gas gun.

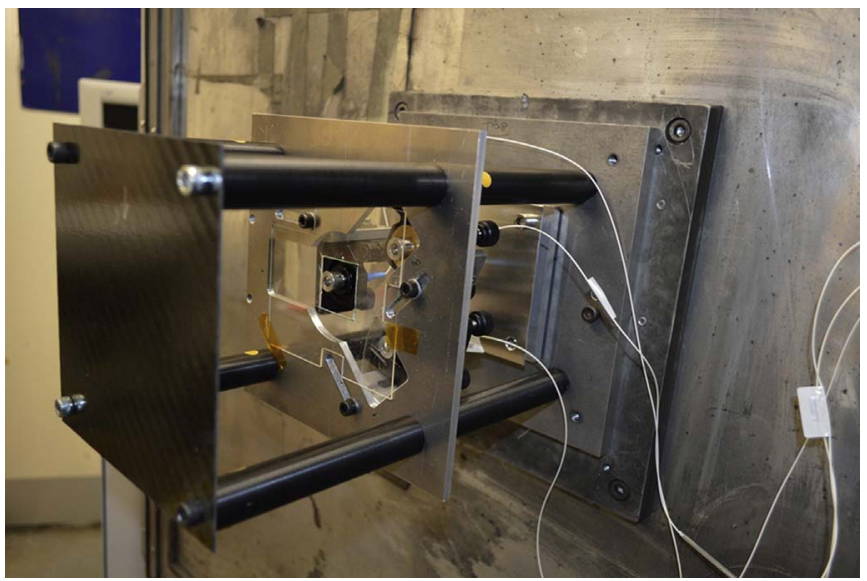

Fig. 8. View of the opened rear port door of the LGG with the fully instrumented target mount, particles coming from the left.

\subsection{Experimental results}

Due to the high running costs of the LGG we were restricted to the number of shots allocated to demonstrate the system. Steel ball bearings of $1 \mathrm{~mm}$ in diameter and $0.004 \mathrm{~g}$ mass were used for shots at $2.11 \mathrm{~km} / \mathrm{s}$ and $4.21 \mathrm{~km} / \mathrm{s}$. The targets are carbon fiber plates $1.5 \mathrm{~mm}$ thick, $15 \mathrm{~cm}$ square, supported on 4 corners.

The results of the impacts measured simultaneously at 3 locations on the rear face of the targets for a shot speed of $2.11 \mathrm{~km} / \mathrm{s}$ (first shot) 
a

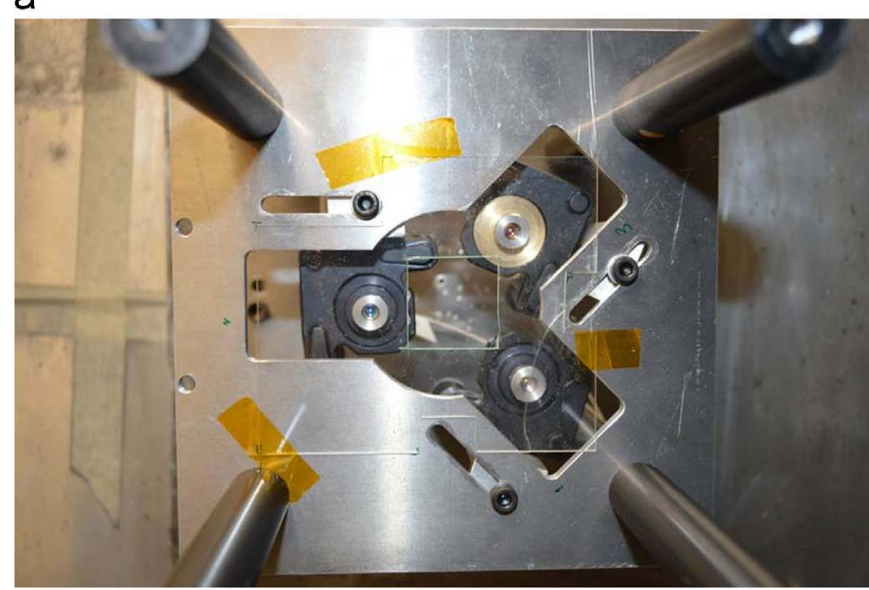

b

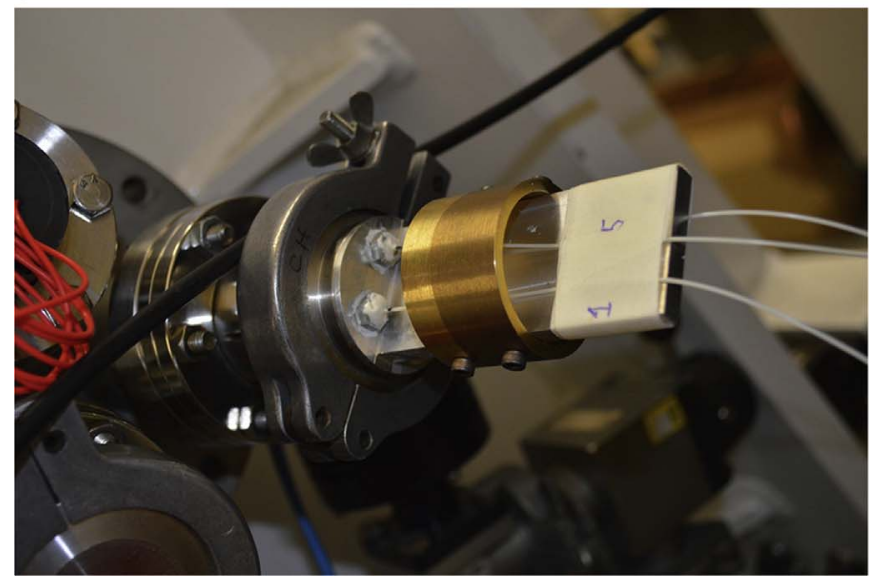

Fig. 9. Details of the installation in the LGG; (a) Installation of the three non-contact probes in the chamber; target removed, particle direction into the paper; (b) Accessing of the fiber leads at the outside of the LGG chamber.

are shown in Fig. 10.

The spectra of the unprocessed signals caused by the impact measured at T1, T2 and T3, shown in Fig. 10a, indicates the excellent performance of the system. The FFT shows a maximum resonance peak found in $295.6 \mathrm{~Hz}$. Other small peaks were also observed indicating the complex low level movements of the plate. Excellent synchronization between the 3 channels was determined with the processing unit. In order to obtain the phase shift among the measured signals, the crosscorrelation was calculated. The results show up to $6.5 \mathrm{mrad}$ of phase shift in all cases $(\sim 3.66 \mu \mathrm{s})$ for the fundamental frequency. Fig. 11 shows the results of simultaneous measurements at two points on the rear face of the target caused by the impact of a steel pellet at a speed of $4.21 \mathrm{~km} / \mathrm{s}$ (second shot). The fiber lead of channel 2 was broken during the measurement due to being hit by debris released during the impact on the plate.

The spectra of Fig. 11a again shown that the optical system functioned well in the very harsh environment of the LGG caused as a result of the impact, which in this case released a large number of fast moving shards of carbon fiber from the target breaking the fiber lead of channel 2. The FFT shown in Fig. 11b of this data again indicates the complexity of the surface vibrations of the target with the maximum resonance peak, in this case, of $281.1 \mathrm{~Hz}$.

\section{Potential applications}

The experimental results shown in previous sections indicate that the system is useful for high and low speed impacts and vibrations. The a

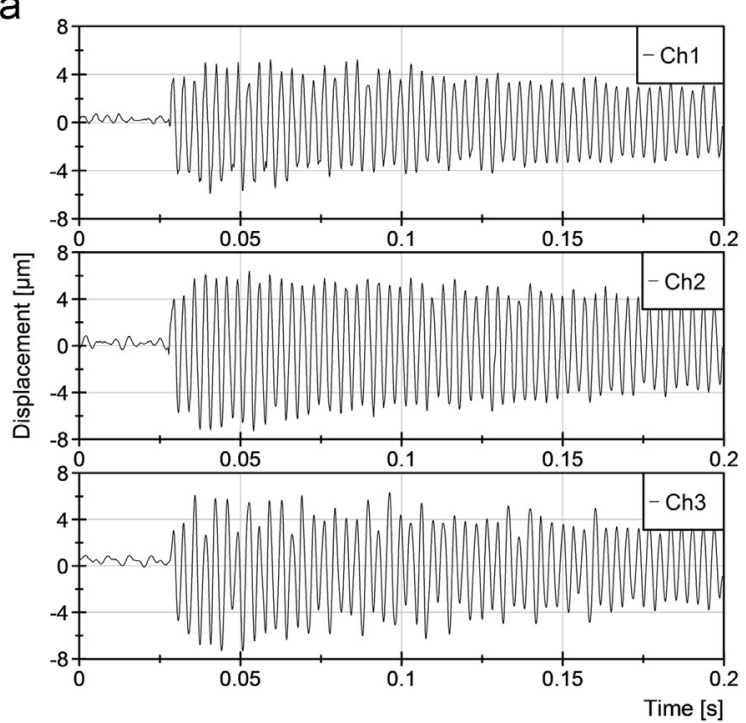

b

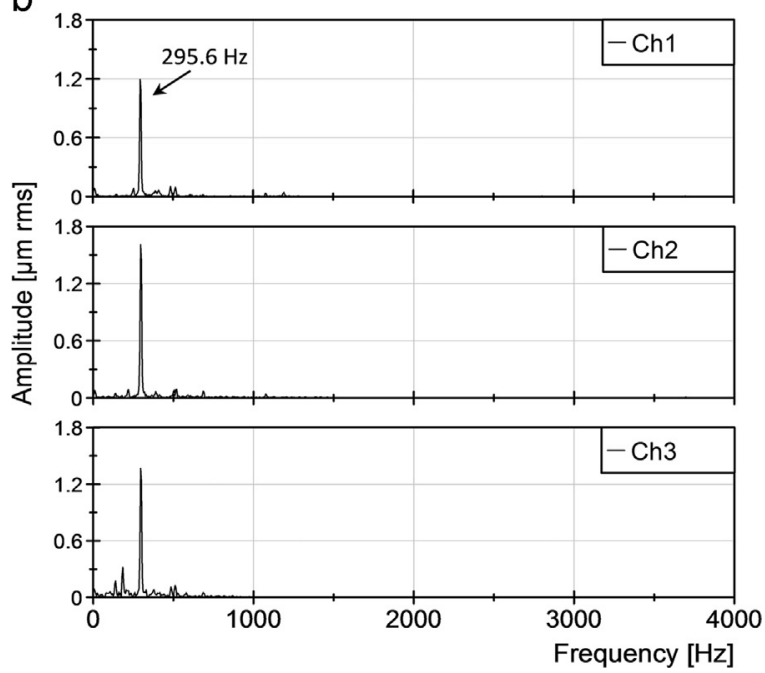

Fig. 10. Measurements of hypervelocity impacts (1st Shot $2.11 \mathrm{~km} / \mathrm{s}$ ) with 3 channels of the MFLDV in the LGG; (a) Raw data taken at 3 locations on rear of target; (b) FFT of the output signals corresponding to the traces shown in (a). The fundamental frequency is $295.6 \mathrm{~Hz}$.

detection of biosignals such as ocular pulse, breathing and heartbeat, among others, are potential applications for MFLDV measuring slow vibrations and impacts. As an example, laser Doppler vibrometry demonstrated that skin vibrations due to the forcing excitation from the heart can be used in imaging blood flow [13]. Moreover, the flexibility of MFLDV to measure vibrations and impacts at inaccessible locations on a target has yet to be explored. For example, in-vivo measurement of teeth mobility [14] is an application in which this unique characteristic of MFLDV can be exploited.

High velocity impact research is mainly directed towards aerospace structures. Due to the problems making measurements and the complexity of the setups, simulations are commonly performed for the study of such structures. Examples of these studies are the linear vibration of wings and controls [15] and the vibrations due to non-linear supersonic panel flutter [16]. Flutter is a very serious problem, especially for modern fighter aircraft. Simulation tools help to design the structure free of flutter, but the only definitive method to clear the aircraft of flutter is flight testing [17]. In this case, the data is usually collected by using conventional accelerometers [18]. MFLDV could give a great advantage for this application, providing high resolution vibration measurements at different points. 


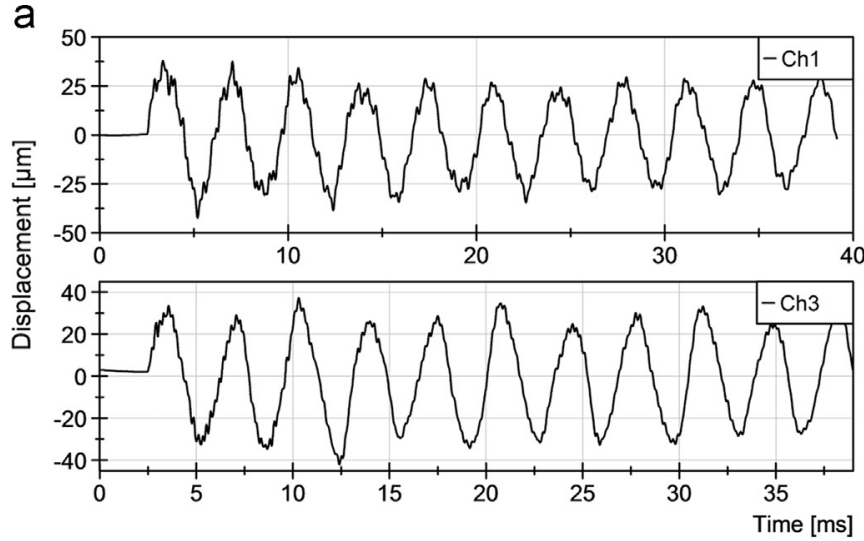

b

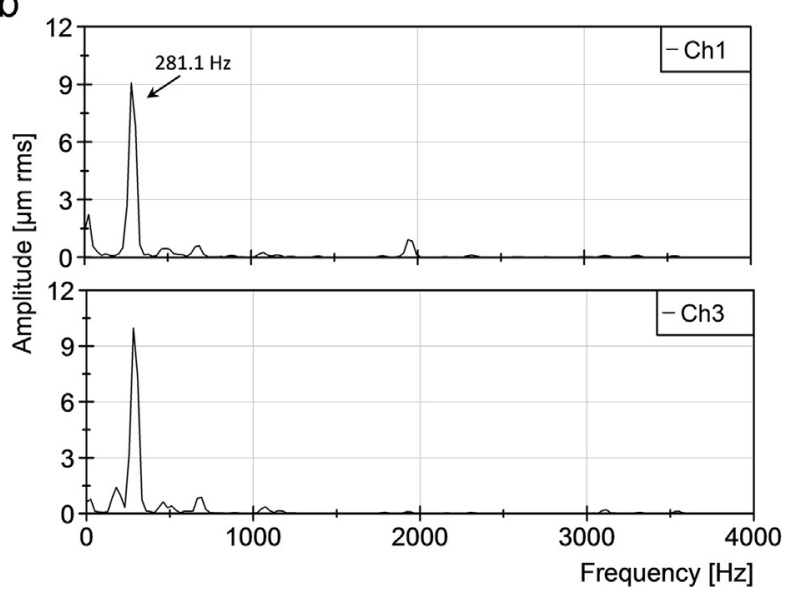

Fig. 11. Measurements of hypervelocity impacts (2nd Shot $4.2 \mathrm{~km} / \mathrm{s}$ ) with 2 channels of the MFLDV in the LGG; (a) Raw data taken at 2 locations (T1 and T3) on rear of target; (b) FFT of the output signals corresponding to the traces in (a). The fundamental frequency is $281.1 \mathrm{~Hz}$.

The versatility of MFLDV enables its installation in other experimental setups for high velocity impact studies. It could be installed in a LGG for studying hypervelocity impacts with metallic particles, as it is reported here, but also in other impact setups of high velocity with complex projectiles, such as ice impacts on woven carbon/epoxy composite panels [19].

\section{Conclusions}

The feasibility of using a MFLDV to make precision non contact vibration measurements over a wide range of velocities (up to $3.8 \mathrm{~m} / \mathrm{s}$ ) has been demonstrated. The use of a digital processing to recover the data enabled the phase, frequency and vibration amplitude to be determined at high resolution. It is feasible to design a relatively small robust portable instrument package containing all the optics and electronics except for the optical fiber transceiver links. This unit when combined with the flexible fibers links with miniature collimators, could be deployed in the applications outlined above. A key application would be for mobile platforms such as Mach 2 jets or the next generation of large passenger planes where simultaneous measurements of impacts on wing/stabilizer could be made for the first time in flight. In addition, it is possible to increase the number of channels to 8 or16 enhancing the number of locations where the effects of impacts or vibration could be simultaneously measured with $1 \mathrm{~mW}$ of optical power per channel.

\section{Acknowledgements}

The authors gratefully acknowledge Prof. M. Burchell for allowing us access to the LGG. Dr. J. E. Posada acknowledges a short term mobility Grant "Ayudas a la movilidad de investigadores - Modalidad B" from University Carlos III de Madrid to undertake research at the University of Kent enabling Dr. Julio E. Posada-Roman to make a significant contribution to the project. The carbon fiber plates were supplied by Dr John Nixon of Advanced Composites.

\section{References}

[1] Abrate S. Modeling of impacts on composite structures. Compos Struct 2001:51:129-38. http://dx.doi.org/10.1016/S0263-8223(00)00138-0.

[2] Dung C, Sasaki E. Numerical simulation of output response of PVDF sensor attached on a cantilever beam subjected to impact loading. Sensors 2016;16:601. http://dx. doi.org $/ 10.3390 / \mathrm{s} 16050601$.

[3] Jia Y, Chen X, Ni Q, Li L, Ju C. Dependence of the impact response of polyvinylidene fluoride sensors on their supporting materials' elasticity. Sensors 2013;13:8669-78. http://dx.doi.org/10.3390/s13070866.

[4] Jackson DA, Cole MJ. Fiber optic interrogation systems for hypervelocity and low velocity impact studies. Photonic Sens 2012;2:50-9. http://dx.doi.org/10.1007/ s13320-011-0040-5.

[5] Burchell MJ, Cole MJ, McDonnell JAM, Zarnecki JC. Hypervelocity impact studies using the $2 \mathrm{MV}$ Van de Graaff accelerator and two-stage light gas gun of the University of Kent at Canterbury. Meas Sci Technol 1999;10:41-50. http://dx.doi. org/10.1088/0957-0233/10/1/011.

[6] Burchell MJ, Standen S, Cole MJ, Corsaro RD, Giovane F, Liou JC, et al. Acoustic response of aluminium and duroid plates to hypervelocity impacts. Int J Impact Eng 2011;38:426-33. http://dx.doi.org/10.1016/j.ijimpeng.2010.10.012.

[7] Kearsley AT, Burchell MJ, Price MC, Graham GA, Wozniakiewicz PJ, Cole MJ, et al Interpretation of Wild 2 dust fine structure: comparison of stardust aluminum foil craters to the three-dimensional shape of experimental impacts by artificial aggregate particles and meteorite powders. Meteorit Planet Sci 2010;44:1489-509. http://dx.doi.org/10.1111/j.1945-5100.2009.tb01188.x.

[8] Posada-Roman JE, Jackson DA, Garcia-Souto JA. Variable configuration fiber optic laser doppler vibrometer system. Photonic Sens 2016;6:97-106. http://dx.doi.org/ 10.1007/s13320-016-0293-0.

[9] Yang C, Guo M, Liu H, Yan K, Xu YJ, Miao H, et al. A multi-point laser Doppler vibrometer with fiber-based configuration. Rev Sci Instrum 2013;84:121702. http://dx.doi.org/10.1063/1.4845335.

[10] Posada-Roman JE, Garcia-Souto JA, Rubio-Serrano J. Multichannel optical-fibre heterodyne interferometer for ultrasound detection of partial discharges in power transformers. Meas Sci Technol 2013;24:94015. http://dx.doi.org/10.1088/09570233/24/9/094015.

[11] Jackson DA, Posada-Roman JE, Garcia-Souto JA. Calibration of laser Doppler vibrometer exploiting Bessel functions of the first kind. Electron Lett 2015;51:1100-2. http://dx.doi.org/10.1049/el.2015.0972.

[12] Di Giovanni M. Flat and corrugated diaphragm design handbook. New York: Marcel Dekker Inc; 1982.

[13] Tabatabai H, Oliver DE, Rohrbaugh JW, Papadopoulos C. Novel applications of laser Doppler vibration measurements to medical imaging. Sens Imaging Int J 2013;14:13-28. http://dx.doi.org/10.1007/s11220-013-0077-1.

[14] Castellini P, Scalise L, Tomasini EP. Teeth mobility measurement: a laser vibrometry approach. J Clin Laser Med Surg 1998;16:269-72. http://dx.doi.org/10. 1089/clm.1998.16.269.

[15] Hao YX, Zhang W, Li SB, Zhang JH. Nonlinear vibrations of FGM cylindrical panel with simply supported edges in air flow. Int J Aerosp Eng 2015;2015:1-14. http:// dx.doi.org/10.1155/2015/246352.

[16] Guo X, Mei C. Application of aeroelastic modes on nonlinear supersonic panel flutter at elevated temperatures. Comput Struct 2006;84:1619-28. http://dx.doi. org/10.1016/j.compstruc.2006.01.041.

[17] Kehoe MW. A historical overview of flight flutter testing. AGARD Struct. Mater Panel Meet., Rotterdam, Netherlands; 1995, p. 1-17.

[18] Corda S, Franz RJ, Blanton JN, Vachon MJ, DeBoer JB. In-flight vibration environment of the NASA F-15B flight test fixture; 2002.

[19] Kim H, Welch DA, Kedward KT. Experimental investigation of high velocity ice impacts on woven carbon/epoxy composite panels. Compos Part A Appl Sci Manuf 2003;34:25-41. http://dx.doi.org/10.1016/S1359-835X(02)00258-0.

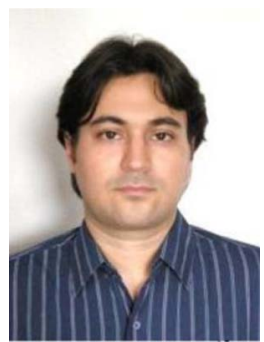

Julio E. Posada-Román received his M.Sc. degree in advanced electronics systems from the Universidad Carlos III de Madrid (UC3M) in 2012 and his Ph.D. in electrical engineering, electronics and automation in 2015 from the same university, where he worked on fiber-optic interferometric sensors for ultrasound measurements and partial discharges acoustic detection in power transformers. He is currently a postdoctoral researcher in the Electronics Technology Department of UC3M, within the Optoelectronics and Laser Technology Group. His research interests are optoelectronic instrumentation systems and fiber optic sensors. 


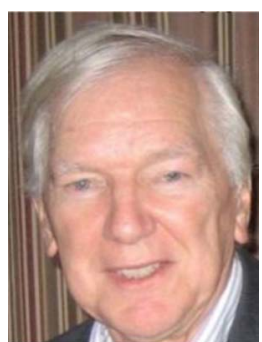

David Jackson has a Ph.D. in nuclear physics from the University of London. He is currently Emeritus Professor of Applied Optics University of Kent UK. He is responsible for many important innovations in the field of optical sensing including laser Doppler velocimetry, non-contact vibration and displacement measurements and fiber optic sensors. His interests in fiber optic sensors include intrinsic and extrinsic single mode optic sensors based upon optical interferometers and fiber Bragg gratings. He has researched optics for medical applications such as OCT, miniature temperature and pressure probes. He has authored or coauthored over 352 journal and 370 conference papers.

Michael John Cole. Experimental Officer in the Center for Astronomy and Planetary Science of the University of Kent Canterbury, UK., for 28 years. He operates the LGG at the University and assists researchers using the machine. The LGG is used to fire projectiles with velocities between 0.25 and $8.3 \mathrm{~km} / \mathrm{s}$ at various targets, thereby simulating the types of impact experienced in space. He has operated a $2 \mathrm{MV}$ Van de Graaff machine for 13 years, which is also used for space related impact work. He was awarded a degree on Physics from the University of London.

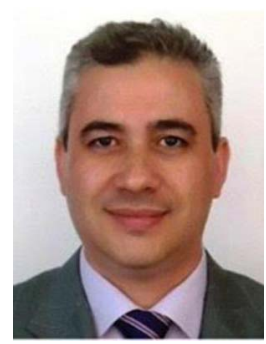

José A. Garcia-Souto was born in Vizcaya, Spain, in 1969. $\mathrm{He}$ received the M.Eng. degree in telecommunication engineering from Universidad Politécnica de Madrid in 1995 and the Ph.D. degree from Universidad Carlos III de Madrid (UC3M) in 2003. He has been an Associate Professor with the Electronics Technology Department, UC3M, since 2004, and a Researcher with the Optoelectronics and Laser Technology Group, Optoelectronic Instrumentation Team. His work has been focused on fiber-optic sensing of acoustic emission and measurements in harsh environments. He has coordinated several research projects in these fields. His current research interests include optical sensors and instrumentation, fiber-optic interferometry and optoelectronics for industrial applications. 